\title{
Electron Transport under the Influence of Two Kinds of Friction in an Electron-Deuteron Plasma
}

\author{
Mitsuaki Nagata \\ Soft Creator Company, Kyoto, Japan \\ Email:nagata@heian-kogyo.jp
}

How to cite this paper: Nagata, M. (2020) Electron Transport under the Influence of Two Kinds of Friction in an ElectronDeuteron Plasma. Journal of Modern Physics, 11, 1751-1760. https://doi.org/10.4236/jmp.2020.1111108

Received: July 20, 2020

Accepted: November 3, 2020

Published: November 6, 2020

Copyright $\odot 2020$ by author(s) and Scientific Research Publishing Inc. This work is licensed under the Creative Commons Attribution International License (CC BY 4.0).

http://creativecommons.org/licenses/by/4.0/

\section{(c) (i) Open Access}

\begin{abstract}
We discuss an electron transport in an ideal plasma which consists of electrons and deuterons. With respect to a frictional force to suppress an unlimited increase of a drift velocity, the Boltzmann equation with the Fokker-Planck collision term takes into consideration only a dynamical frictional force coming from the many-body collisions through the Coulomb force. However, we here bring forward a problem that there may be another frictional force besides the dynamical frictional force. Another frictional force was found in the weakly ionized plasma and appears only in the case where free paths (nearly straight lines in no external force field) can be defined. Then, we have inquired into the existence of physical quantities like free paths (or free times) in the field of the scattering through the Coulomb force and the existence of an effective radius of the Coulomb force of a deuteron.
\end{abstract}

\section{Keywords}

Conductivity of Electrons in a Perfectly Ionized Plasma, Many-Body and Two-Body Collisions, Effective Radius of the Coulomb Force

\section{Introduction}

Electron transport in a fully ionized plasma has been classically analyzed based on the Boltzmann equation with the Fokker-Planck collision term $\delta f_{\mathrm{c}} / \delta t \quad(t$. time) which is given by [1]-[6]

$$
\frac{\delta f_{\mathrm{c}}}{\delta t}=-\frac{\partial}{\partial v_{\mathrm{r}}}\left(f \frac{\partial \mathrm{H}}{\partial v_{\mathrm{r}}}\right)+\frac{1}{2} \frac{\partial^{2}}{\partial v_{\mathrm{r}} \partial v_{\mathrm{s}}}\left(f \frac{\partial^{2} \mathrm{G}}{\partial v_{\mathrm{r}} \partial v_{\mathrm{s}}}\right)
$$

Here, $f$ is a velocity distribution function of electrons, $v_{\mathrm{r}(\mathrm{r}=x, y, z)}$ and $v_{\mathrm{s}(\mathrm{s}=x, y, z)}$ are components of a velocity variable $v$ of an electron, 


$$
\mathrm{H} \simeq \Gamma_{v} \frac{n_{\mathrm{p}}}{|\boldsymbol{v}|}, \mathrm{G} \simeq \Gamma_{v} n_{\mathrm{p}}|\boldsymbol{v}|, \Gamma_{v}=4 \pi\left(\frac{q^{2}}{4 \pi \varepsilon_{0} m_{\mathrm{e}}}\right)^{2}\left(\ln \frac{4 \pi \varepsilon_{0} m_{\mathrm{e}} v^{2} \lambda_{\mathrm{D}}}{q^{2}}\right)
$$

$-q$ is the electron charge $1.6 \times 10^{-19} \mathrm{C}, m_{\mathrm{e}}$ is the rest mass of an electron $9.1 \times$ $10^{-31} \mathrm{~kg}, \quad \varepsilon_{0}$ is the dielectric constant of vacuum $8.855 \times 10^{-12} \mathrm{Farad} / \mathrm{m}, \lambda_{\mathrm{D}}$ is the Debye length $\left(\varepsilon_{0} k_{\mathrm{B}} T / n_{\mathrm{p}} q^{2}\right)^{1 / 2}, n_{\mathrm{p}}$ is a deuteron density, $k_{\mathrm{B}}$ is the Boltzmann constant $1.38 \times 10^{-23} \mathrm{~J} / \mathrm{K}$ and $T$ is electron temperature (=plasma temperature), $m_{\mathrm{e}} \ll m_{\mathrm{p}}$ (deuteron mass) and $|\boldsymbol{v}| \gg\left|\boldsymbol{v}_{\mathrm{p}}\right|$ (deuteron velocity magnitude). By integrating the Boltzmann equation multiplied by $m_{\mathrm{e}} v$ all over the velocity space of electrons, the following equation for a drift velocity (denoted by $\mathbf{u}(t)$ ) is obtained:

$$
m_{\mathrm{e}} \frac{\mathrm{d} \mathbf{u}(t)}{\mathrm{d} t}+q \boldsymbol{E}=\frac{m_{e}}{n_{e}} \int f \frac{\partial \mathrm{H}}{\partial \boldsymbol{v}} \mathrm{d} \boldsymbol{v}
$$

Here, $\boldsymbol{E}$ is an electric field, a magnetic field $\boldsymbol{B}=0, n_{\mathrm{e}}$ is an electron density which is not a function of position. Also, in (2), it has been taken into consideration that the dispersion due to work of the term containing $G$ in (1) leaves the total momentum of the plasma unchanged. Furthermore, products between components of the drift velocity have been neglected. The right-hand side of (2) is the dynamical frictional force [1] (called DFF) coming from the many-body collisions between electrons and lots of surrounding charged particles.

On the other hand, we previously derived the momentum-transfer equation (given in (20) of Ref. [7]) in the case where electrons collide with neutral atoms and ions having an effective radius with respect to the Coulomb force (Determining the effective radius had remained as a pending question in Ref. [7]). As a result, two kinds of the frictional forces against an electric field, a part of DFF and another new frictional force, had appeared. An external electric field truncates or enlarges lengths of free times of electrons in the two-body collisions. We explained theoretically in Appendix of the other Ref. [8] that such a truncation-work of the electric field generates a new frictional force (called TFF) against an electron flow. TFF appears only in the case where a physical quantity like free times can be defined. We bring forward here a problem that, also in a field of scattering through the Coulomb force, there may be both DFF and TFF. In the following Section 2, we describe concretely DFF and TFF for comparison. And in Section 3, we try estimating an effective radius of the Coulomb force of a deuteron in the two-body collisions (called the e- $\mathrm{D}^{+}$collisions).

\section{Two Kinds of the Frictional Force against Electric Field-Acceleration}

First, for simplification of discussion, through this research we set the following physical conditions and the following ideal plasma:

* About external force fields;

(0) An electric field $\boldsymbol{E}=-\hat{\mathrm{z}} \mathrm{E} \mathrm{V} / \mathrm{m}$.

(O) A magnetic field $\boldsymbol{B}=0 \mathrm{~T}$. 
* About an ideal plasma consisting of electrons and deuterons;

(C) Electron density $n_{\mathrm{e}}=10^{21} \mathrm{~m}^{-3}$.

(a) Deuteron density $n_{\mathrm{p}}=n_{e} \mathrm{~m}^{-3}$.

(C) Plasma temperature $T=4 \times 10^{8} \mathrm{~K}$.

(a) A deuteron $\mathrm{D}^{+}$is regarded as a rest, heavy particle to an electron $\left(m \ll m_{\mathrm{p}}\right.$, $\left.|v| \gg\left|v_{\mathrm{p}}\right|\right)$.

(a) Every electron is treated as a nonrelativistic particle with the same velocity $\bar{v} \quad\left(=\right.$ a mean thermal velocity $\left.\left(8 k_{\mathrm{B}} T / \pi m_{\mathrm{e}}\right)^{1 / 2}=1.2 \times 10^{8} \mathrm{~m} / \mathrm{sec}\right)$, except for calculation from (2) to (4).

(O) An influence [2] of the electron-electron interaction on the momentum-transfer from electrons to deuterons is disregarded.

(0) The Ramsauer effect also is disregarded.

1) The frictional force DFF in the field of the many-body collisions: Substituting into (2)

$$
\left\{\begin{array}{l}
f=n_{\mathrm{e}}\left(\frac{m_{\mathrm{e}}}{2 \pi k_{\mathrm{B}} T}\right)^{3 / 2} \exp \left[-\frac{m_{\mathrm{e}}}{2 k_{\mathrm{B}} T}(v-\mathbf{u}(t))^{2}\right] \\
\boldsymbol{E}=-\hat{z} E \text { and } \mathbf{u}(t)=\hat{z} \mathrm{u}(t),
\end{array}\right.
$$

We have [3] [4] in the limit of small $\left(\frac{m}{2 k_{\mathrm{B}} T}\right)^{1 / 2} u(t)$,

$$
m_{\mathrm{e}} \frac{\mathrm{d} u(t)}{\mathrm{d} t}-q E=-m_{\mathrm{e}} v_{\mathrm{D}} \mathrm{u}(t)
$$

where, by using $E_{0}=\left(n_{\mathrm{p}} m_{\mathrm{e}} \Gamma / q\right)\left(2 k_{\mathrm{B}} T / m_{\mathrm{e}}\right), \quad \Gamma=4 \pi\left(\bar{v}^{2}\right)^{2} p_{\perp}^{2} \ln \left(\lambda_{\mathrm{D}} / p_{\perp}\right)$ and $p_{\perp}=q^{2} /\left(4 \pi \varepsilon_{0} m_{\mathrm{e}} \bar{v}^{2}\right) \quad$ (Dreicer [3] used $3 k_{\mathrm{B}} T / \pi m_{\mathrm{e}}$ instead of $\left.\bar{v}^{2}\left(=8 k_{\mathrm{B}} T / \pi m_{\mathrm{e}}\right)\right)$,

$$
v_{\mathrm{D}}=\frac{q E_{0}}{m_{\mathrm{e}}} \frac{4}{3 \pi^{1 / 2}}\left(\frac{m_{\mathrm{e}}}{2 k_{\mathrm{B}} T}\right)^{1 / 2}=n_{\mathrm{p}} \pi p_{\perp}^{2} \bar{v}\left(4 \ln \frac{\lambda_{\mathrm{D}}}{p_{\perp}}\right) \frac{32}{3 \pi^{2}}
$$

Carrying out numerical calculations for $\lambda_{\mathrm{D}}, p_{\perp} v_{\mathrm{D}}$, we have

$$
\begin{aligned}
& \lambda_{\mathrm{D}}=\left(\frac{\varepsilon_{0} k_{\mathrm{B}} T}{n_{\mathrm{p}} q^{2}}\right)^{1 / 2}=4.37 \times 10^{-5} \mathrm{~m} \\
& p_{\perp}=\frac{q^{2}}{4 \pi \varepsilon_{0} m_{\mathrm{e}} \bar{v}^{2}}=1.76 \times 10^{-14} \mathrm{~m}
\end{aligned}
$$

( $p_{\perp}$ is the impact parameter for $\pi / 2$-deflection in the $\mathrm{e}-\mathrm{D}^{+}$collisions)

$$
v_{\mathrm{D}} \simeq 10^{4} \mathrm{sec}^{-1}
$$

The right-hand side of (4) $-m_{\mathrm{e}} v_{\mathrm{D}} \mathrm{u}(t)$ is commonly called DFF.

2) Another frictional force TFF in the field of the e- $D^{+}$two-body collisions:

The momentum-transfer equation in the case where electrons do the two-body collisions both with neutral atoms and with ions is given by (20) of Ref. [7], as mentioned already. The equation reduces as follows, in the cases of no neutral atoms $\left(v^{\text {en }}=0\right)$ and no magnetic field ( $\boldsymbol{B}=0$, 


$$
\begin{aligned}
\left.\boldsymbol{F}_{0} \times \hat{\mathbf{b}}=\left(-q \boldsymbol{E}+m_{\mathrm{e}} \nu^{\mathrm{ep}} \zeta \mathbf{u}(t)\right) \times(\boldsymbol{B} /|\boldsymbol{B}|)=0\right), \\
\quad m_{\mathrm{e}} \frac{\mathrm{d} \mathbf{u}(t)}{\mathrm{d} t}+q \boldsymbol{E}=-m_{\mathrm{e}} \nu^{\mathrm{ep}}(1-\zeta) \mathbf{u}(t)-\kappa\left(-q \boldsymbol{E}+m_{\mathrm{e}} v^{\mathrm{ep}} \zeta \mathbf{u}(t)\right)
\end{aligned}
$$

Here, $v^{\mathrm{ep}}=n_{\mathrm{p}} \pi p^{2} \bar{v}$. In this work, this corresponds to the collision frequency of electrons in the e- $\mathrm{D}^{+}$collisions, and $p$ is an effective radius of the Coulomb force of $\mathrm{D}^{+}$. Determining a value of $p$ had been remained as the pending question, $\zeta$ is the remaining ratio of $\mathbf{u}(t)$ in the e- $\mathrm{D}^{+}$anisotropic collisions (shown in Figure 1 of Ref. [7]) and is given by

$$
1-\zeta=4 \frac{\ln \Lambda}{\Lambda^{2}}
$$

(where, $\Lambda=p / p_{\perp}$. In the isotropic scattering, $\zeta=0$ ),

$$
\kappa \equiv \beta_{02\left(\omega_{\mathrm{c}}=0\right)}=\frac{1}{3}
$$

(where, $\left.\omega_{\mathrm{c}}=q|\boldsymbol{B}| / m_{\mathrm{e}}\right)$, and $-\kappa\left(-q \boldsymbol{E}+m_{\mathrm{e}} \nu^{\mathrm{ep}} \zeta \mathbf{u}(t)\right)$ is TFF. The quantity $v^{\mathrm{ep}}(1-\zeta)$ with $p=\lambda_{D}$ is the same with $v_{\mathrm{D}}$ of (5) in structure. Therefore, DFF $\left(=-m_{\mathrm{e}} \nu_{\mathrm{D}} \mathbf{u}(t)\right.$ of $\left.(4)\right)$ contains $-m_{\mathrm{e}} \nu^{\mathrm{ep}}(1-\zeta) \mathbf{u}(t)$ as a part of itself. We presume that both DFF and TFF will work to suppress an unlimited increase of a drift velocity by acceleration of an external electric field.

\section{An Effective Radius of a Deuteron on the Coulomb Force Scattering}

We try estimating the upper limit of an impact parameter $p$ of $v^{\text {ep }}$ in (7). Since

C-sphere

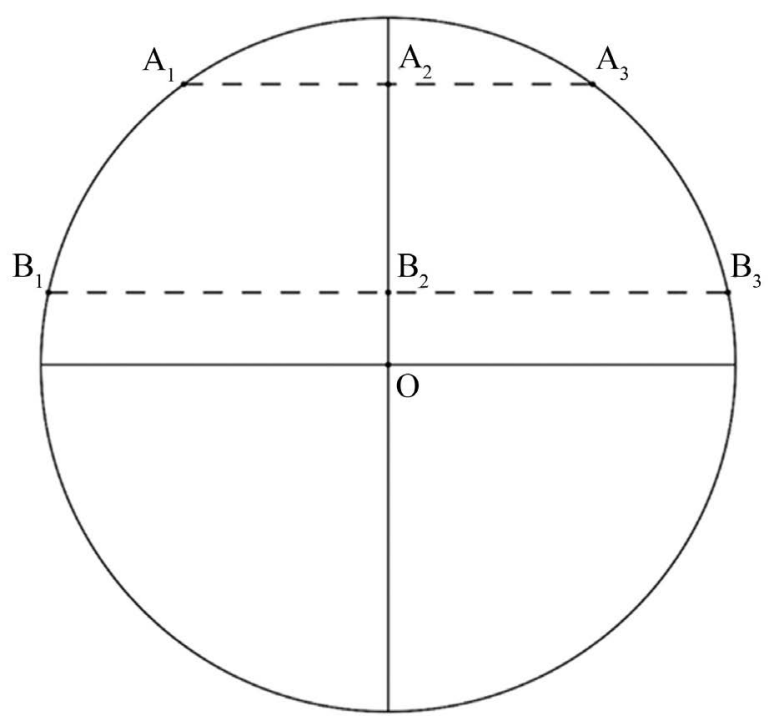

Figure 1. A proper influence-sphere ( $C$-sphere), with respect to the Coulomb force, of a deuteron being at point $\mathrm{O}$. The radius of $C$-sphere is $0.5 d$. An electron flies two paths (from $\mathrm{A}_{1}$ to around $\mathrm{A}_{3}$ and from $\mathrm{B}_{1}$ to around $\mathrm{B}_{3}$ ). $\overline{\mathrm{OA}_{2}}=0.4 d, \overline{\mathrm{OB}_{2}}=0.1 d$, $\overline{\mathrm{A}_{1} \mathrm{~A}_{2}}=\overline{\mathrm{A}_{2} \mathrm{~A}_{3}} \simeq 0.33 d, \overline{\mathrm{B}_{1} \mathrm{~B}_{2}}=\overline{\mathrm{B}_{2} \mathrm{~B}_{3}} \simeq 0.5 d . d$ is a mean distance between deuterons. 
a mean distance between deuterons is $n_{\mathrm{p}}^{-1 / 3}\left(=d=10^{-7} \mathrm{~m}\right)$, a deuteron has a proper influence-sphere (called $C$-sphere) whose radius is $0.5 d$ with respect the Coulomb force [9]. We first compare deflection-angles by the many-body collisions with the ones by the two-body collisions in the cases where an electron passes the inside of $C$-sphere. In Figure 1 , a deuteron $\mathrm{D}^{+}$is at point $\mathrm{O}$ and an electron with $\bar{v}\left(=1.2 \times 10^{8} \mathrm{~m} / \mathrm{sec}\right)$ flies two paths (from point $\mathrm{A}_{1}$ to around point $A_{3}$ and from point $B_{1}$ to around point $B_{3}$ ) under no external force fields.

1) The case of the many-body collisions:

The magnitude of the dynamical frictional coefficient $\Delta v_{\|}$in the Fokker-Planck collision term is given by

$$
\Delta v_{\|} t=n_{\mathrm{p}}\left[4 \pi \bar{v}^{2} p_{\perp}^{2} \ln \left(\frac{\lambda_{\mathrm{D}}}{P_{\perp}}\right)\right] t=1.21 \times 10^{12} t \quad \mathrm{~m} / \mathrm{sec}
$$

where $t$ is flight time. Also, the dispersion coefficient $\Delta v_{\perp}$ is given by

$$
\Delta v_{\perp} t \simeq\left(2 \bar{v} \Delta v_{\|} t\right)^{1 / 2}=1.71 \times 10^{10} t^{1 / 2} \mathrm{~m} / \mathrm{sec} \quad\left(\Delta v_{\|} t \ll \bar{v}\right)
$$

When an electron flies from $\mathrm{A}_{1}$ to around point $\mathrm{A}_{3}(t \simeq(2 d / 3) / \bar{v})$,

$$
\begin{gathered}
\Delta v_{\|} t=0.67 \times 10^{-3} \mathrm{~m} / \mathrm{sec} \quad\left(\text { Note: } \Delta v_{\|} t \ll \bar{v}\right) . \\
\Delta v_{\perp} t=4 \times 10^{2} \mathrm{~m} / \mathrm{sec}
\end{gathered}
$$

(Effects of $\Delta v_{\|} t$ on movement of an electron is disregarded in the after discussion).

Accordingly, a deflection angle $\chi_{\text {many }}$ after the flight is

$$
\chi_{\text {many }\left(\mathrm{A}_{1} \rightarrow \mathrm{A}_{3}\right)} \simeq \frac{\Delta v_{\perp} t}{\bar{v}}=33.3 \times 10^{-7} \text { radian }
$$

2) The case of the e- $D^{+}$collisions:

We denote deflection-angles of an electron for two impact parameters $\left(\overline{\mathrm{OA}_{2}}=0.4 d, \overline{\mathrm{OB}_{2}}=0.1 d\right)$ by $\chi_{\mathrm{t} 1}, \chi_{\mathrm{t} 2}$, respectively.

a) $\overline{\mathrm{OA}_{2}}=0.4 d \mathrm{~m}$. Since $p$ and $l \mathrm{in}$ (A3) of Appendix are $0.4 d$, about $0.33 d$, respectively, we have

$$
\tan \frac{\chi_{\mathrm{t} 1}}{2}=\frac{p_{\perp}}{0.4 d} \cdot \frac{0.33 d}{\left[(0.33 d)^{2}+(0.4 d)^{2}\right]^{1 / 2}} \therefore \chi_{\mathrm{t} 1} \simeq 5.64 \times 10^{-7} \text { radian }
$$

b) $\overline{\mathrm{OB}_{2}}=0.1 d \mathrm{~m}$. Since $l \simeq 0.5 d, \chi_{\mathrm{t} 2} \simeq 36 \times 10^{-7}$ radian .

Now, we aim an electron (called Test electron) which starts at time $t=0$ from some point of a real plasma space. A scope of probable positions where Test electron is to exist at time $t$ expands from a dot to a tiny sphere (being supposed to be a round sphere and called $P$-sphere) due to the dispersion in a velocity space by the many-body collisions. Based on the dispersion given in (9), calculating a radius $P_{48 d}$ of $P$-sphere after it has flied, for instance, the length $48 d$, we have

$$
P_{48 d}=\int_{0}^{\frac{48 d}{\bar{v}}} \Delta v_{\perp} t \mathrm{~d} t=0.91 \times 10^{-10} \mathrm{~m}
$$


Since $d \gg P_{48 d}, P$-sphere can be regarded to be a tiny particle with minus electric charge and an expanding volume. We analyze this orbit of the length $48 d$ as follows:

1) The orbit of $P$-sphere consists of paths (called paths-in) which pass insides of $C$-spheres and paths (called paths-out) which do not pass the insides of $C$-spheres.

2) In paths-out, the dispersion makes a volume of $P$-sphere expand but does not make an advance-direction of $P$-sphere change.

3) In paths-in, the attraction by a deuteron at the center of each $C$-sphere gives proper, strong influence upon $P$-sphere. Because of this influence, " $P$-sphere suffers a small change of the advance-direction”. However, unless $P$-sphere approaches a deuteron with too small impact parameters, the magnitude of the direction-changes are very small, as seen in the values of $\chi_{\mathrm{t} 1}, \chi_{\mathrm{t} 2}$ of (12), (13) and $\chi$ of (16). Also, since the direction-changes are done in disorder, we presume that $P$-sphere will advance straight macroscopically, although it is accompanied by small meanders.

4) As mentioned above, we have introduced a new element that the orbit of $P$-sphere is classified into straight parts in paths-out and the meandering parts in paths-in. The dynamical frictional coefficient decreases the speed of $P$-sphere and the dispersion coefficient makes the volume of $P$-sphere expand, but the above two coefficients do not make the advance-direction change. However, we consider that, when $P$-sphere (a tiny particle) goes deep a little into the inside of $C$-sphere, the orbit of $P$-sphere ought to suffer some fluctuation (or meander). The orbital meander in a real plasma space gives mainly swinging-motions to the velocity-point corresponding to the central point of $P$-sphere, besides the small movement by the dynamical frictional force. However, as mentioned in the above (3), small disorderly swinging-motions in the velocity-space does not obstruct that $P$-sphere advances straight macroscopically.

5) We estimate a mean length $\left(=\lambda_{\text {out }}\right)$ of paths-out. If $C$-sphere is a solid sphere, $\lambda_{\text {out }}$ is given by $1 /\left(n_{\mathrm{p}} \pi d^{2} / 4\right)=4 d / \pi=1.27 d$. But since plural $C$-spheres can hold the same space in common and furthermore since projections of $C$-spheres which are seen from $P$-sphere will overlap because of the big volume of $C$-sphere, we assume roughly that $\lambda_{\text {out }} \simeq 2.5 d$.

Let us consider the case where $P$-sphere continues to fly over the length $48 d$ and has come around point $\mathrm{O}_{1}$ shown in Figure 2. This point is inside of $P$-sphere and Test electron is at this point. Figure 2 shows an imaginary sight that the volume of a new probable positions-sphere of Test electron starting from point $\mathrm{O}_{1}$ gradually expands. The new probable positions-sphere arrives at point $\mathrm{O}_{2}$ on the surface of a $C$-sphere after having flied the length $\lambda_{\text {out }}$ without suffering meander and is about to make a head-on collision with a deuteron nucleus being at point $\mathrm{O}_{3}$. The central point of the new probable positions-sphere does not suffer any meander in the head-on collision path from $\mathrm{O}_{2}$ to $\mathrm{O}_{3}$. Calculating a radius $\left(=p_{\text {up }}\right)$ of the new probable positions-sphere near point $\mathrm{O}_{3}$, we have 


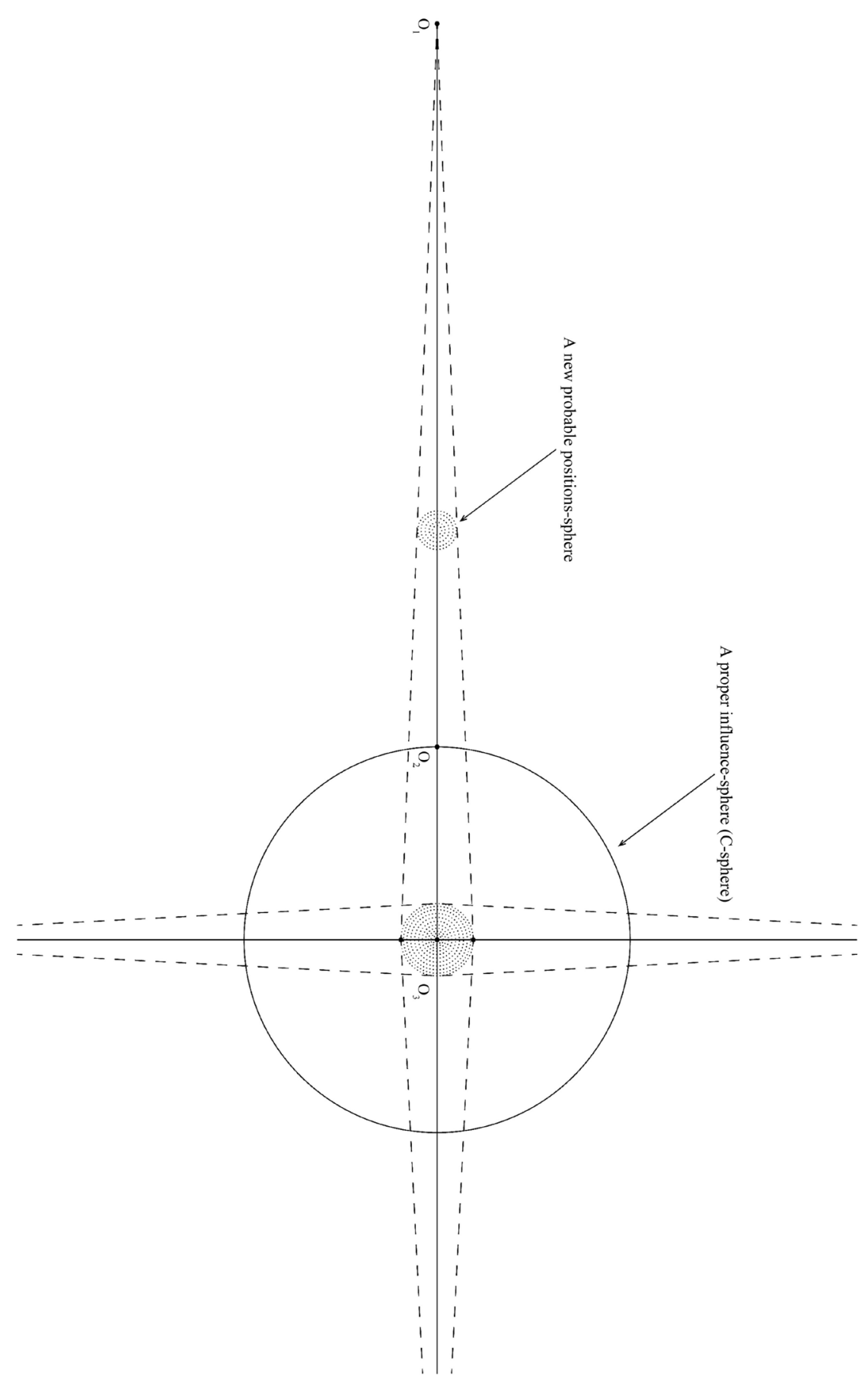

Figure 2. Expansion of a new probable positions-sphere of an electron by the dispersion. The central point of a new probable positions-sphere starts from point $\mathrm{O}_{1}$ and makes a head-on collision with a deuteron nucleus being at point $\mathrm{O}_{3} \cdot \overline{\mathrm{O}_{1} \mathrm{O}_{2}}=2.5 \mathrm{~d}$ and $\overline{\mathrm{O}_{2} \mathrm{O}_{3}}=0.5 d$.

$$
p_{\text {up }}=\int_{0}^{\frac{2.5 d+0.5 d}{\bar{v}}} \Delta v_{\perp} t \mathrm{~d} t=\frac{2}{3} \times 1.71 \times 10^{10}\left(\frac{3 d}{\bar{v}}\right)^{3 / 2}=1.43 \times 10^{-12}=81 p_{\perp} m
$$

The above $p_{\mathrm{up}}$ is the function of $\left(\overline{\mathrm{O}_{1} \mathrm{O}_{3}}\right)^{3 / 2}$. Considering that most of lengths 
of paths-out is in the range between $d$ and $4 d$ and calculating $p_{\text {up }}$ in two cases where $\overline{\mathrm{O}_{1} \mathrm{O}_{3}}=d+0.5 d$ and $\overline{\mathrm{O}_{1} \mathrm{O}_{3}}=4 d+0.5 d$, we have

$$
\left\{\begin{array}{l}
p_{\text {up }}=81 p_{\perp} \times 0.35 \text { for } \overline{\mathrm{O}_{1} \mathrm{O}_{3}}=3 d \times 0.5 \\
p_{\text {up }}=81 p_{\perp} \times 1.84 \text { for } \overline{\mathrm{O}_{1} \mathrm{O}_{3}}=3 d \times 1.5
\end{array}\right.
$$

Though a mean length of paths-out depends also on a radius of $C$-sphere, now, we set an upper limit of an impact parameter for the e- $\mathrm{D}^{+}$two-body collisions to (15). A deflection-angle $\chi$ corresponding to $p_{\mathrm{up}}$ of (15) is, from $\tan \chi / 2 \simeq p_{\perp} / p_{\text {up }}$,

$$
\chi=1.4 \text { degree }
$$

Since a sphere with the radius $p_{\text {up }}$ is little tiny, we assume that $v^{\text {ep }}$ in (4) is $n_{\mathrm{p}} \pi p_{\mathrm{up}}^{2} \bar{v}$. Then,

$$
\begin{gathered}
v^{\text {ep }}=7.65 \times 10^{5} \mathrm{sec}^{-1}, 1-\zeta=4\left(\frac{p_{\perp}}{p_{\text {up }}}\right)^{2} \ln \left(\frac{p_{\text {up }}}{p_{\perp}}\right)=2.7 \times 10^{-3} \\
\zeta=0.9973, \quad v^{\text {ep }}(1-\zeta)=0.21 \times 10^{4} \mathrm{sec}^{-1}
\end{gathered}
$$

$\left(v_{\mathrm{D}}\right)^{-1}$ is the characteristic time to change plasma conditions through the many-body collisions. On the other hand, since $\left(v^{\text {ep }}\right)^{-1}=\left(v_{\mathrm{D}}\right)^{-1} / 77$, this time $\left(v^{\text {ep }}\right)^{-1}$ is too short for the dispersion to play an important role.

Now, asking for the drift velocity $u$ (the steady-state solution), we have the following results:

1) From (4);

$$
\mathrm{u}=\mathrm{u}_{\text {many }}=\frac{q E}{m_{\mathrm{e}}} \frac{1}{v_{\mathrm{D}}} \simeq 1.76 \times 10^{7} E \mathrm{~m} / \mathrm{sec}
$$

If the influence of the electron-electron scattering [2] is taken into consideration as the frictional force,

$$
\mathrm{u}=\mathrm{u}_{\text {many }} \simeq 1.76 \times 10^{7} \times 0.58 E=10^{7} E \mathrm{~m} / \mathrm{sec}
$$

2) From (7);

$$
\mathrm{u}=\mathrm{u}_{\mathrm{two}}=\frac{q E}{m_{\mathrm{e}}} \frac{1-\kappa}{v^{\mathrm{ep}}(1-\zeta)+\kappa v^{\mathrm{ep}} \zeta}=4.57 \times 10^{5} E=\frac{\mathrm{u}_{\text {many }}}{38.5} \mathrm{~m} / \mathrm{sec}
$$

The quantity $-m_{\mathrm{e}} v^{\mathrm{ep}}(1-\zeta) \mathrm{u}(t)$ of $(7)$ is a part of the dynamical frictional force, as already mentioned. Then, if $v^{\text {ep }}(1-\zeta)$ of $(20)$ is replaced with $v_{\mathrm{D}}$, $\mathrm{u}_{\text {two }}$ becomes $4.4 \times 10^{5} \mathrm{Em} / \mathrm{sec}$. The dynamical frictional force may be too weak to suppress "the runaway instability". If the truncation frictional force works, the fear of outbreak of the runaway instability is somewhat lightened.

\section{Conclusions}

It is noted that the extension of positions of an electron in a real plasma space due to both the dispersion and the dynamical friction defined in a velocity-space is very small. The extension-region has been roughly regarded as a tiny sphere whose volume expands with time. The tiny sphere advances straight statistically 
in no external force fields. But we have introduced the new element that the tiny sphere suffers fluctuation (or meander) near each deuteron and have imagined that tiny spheres starting from various positions make head-on collisions with a deuteron nucleus straight without suffering meander. Finally we put in order the consideration on the estimation of an effective radius of the Coulomb force of a deuteron:

1) There ought to be the mean length of paths by which a tiny sphere advances straight without suffering meander.

2) We set a Sphere with the radius of the mean length plus a half of the mean distance between deuterons. A deuteron is at the center of Sphere.

3) From every point on the surface of Sphere, each electron starts toward the deuteron at the same time.

4) Those electrons will form a cloud around the deuteron nucleus. We consider the cloud to be an effective region (for the two-body interaction) of the Coulomb force of a deuteron.

\section{Conflicts of Interest}

The author declares no conflicts of interest regarding the publication of this paper.

\section{References}

[1] Chandrasekhar, S. (1943) Astrophysical Journal, 97, 255. https://doi.org/10.1086/144517

[2] Spitzer, L. and Härm, A. (1953) Physical Review Journals Archive, 89, 997. https://doi.org/10.1103/PhysRev.89.977

[3] Dreicer, H. (1959) Physical Review Journals Archive, 115, 238. https://doi.org/10.1103/PhysRev.115.238

[4] Linhart, J.G. (1961) Plasma Physics. 2nd Edition, North-Holland Publishing Co., Netherlands.

[5] Moffat, W.C. (1961) Thermodynamic and Electrical Properties of Dissociated Combustion Gases. MIT Magneto Gas Dynamic Lab. Reptort 5.

[6] Baral, K.C. and Mohanty, J.N. (1997) Physics of Plasmas, 4, 2010. https://doi.org/10.1063/1.872396

[7] Nagata, M. (2011) The European Physical Journal D, 65, 429-440. https://doi.org/10.1140/epjd/e2011-10464-2

[8] Nagata, M. (1999) Journal of the Physical Society of Japan, 68, 2281-2286. https://doi.org/10.1143/JPSJ.68.2281

[9] Conwell, E. and Weisskoph, V. E. (1946) Physical Review Journals Archive, 69, 258A.

[10] Landau, L. and Lifshitz, E. (1987) The Classical Theory of Fields. Sec. 38, 4th Revised English Edition, Butterworth \& Heinemann, Amsterdam, 99. 


\section{Appendix}

We ask for a relation between a deflection angle $\chi$ and an impact parameter $p$ in the case where a relativistic electron with the velocity $\bar{v}$ is deflected by an electric field of a deuteron $\mathrm{D}^{+}$. The deuteron $\mathrm{D}^{+}$is at the origin $x=y=0$. An electron with the velocity $\bar{v}$ and the mass $m_{\mathrm{er}}\left(=m_{\mathrm{e}} /\left(1-\bar{v}^{2} / c^{2}\right)^{1 / 2} \equiv m_{\mathrm{e}} / \gamma_{\mathrm{r}}\right)$ approaches the ion $\mathrm{D}^{+}$along the $x$-axis. A position $\mathbf{r}(t)$ of the electron at time $t$ is

$$
\mathbf{r}(t)=\hat{x} \bar{v} t+\hat{y} p\left(-\frac{l}{\bar{v}}<t \leq 0\right)
$$

Variations of the velocity and the mass changing with time during the interaction with $\mathrm{D}^{+}$are disregarded. An electric field which the electron flying with the constant velocity $\bar{v}$ feels is given by [10]

$$
\mathbf{E}\left(t^{\prime \prime}\right)=\frac{q \gamma_{r}^{2} \mathbf{r}(t)}{4 \pi \varepsilon_{0}\left[(\bar{v} t)^{2}+\gamma_{r}^{2} p^{2}\right]^{3 / 2}} \equiv \hat{x} E_{\|}^{\prime \prime}+\hat{y} E_{\perp}^{\prime \prime}
$$

Then, a deflection angle $\chi$ of the electron by the deuteron $\mathrm{D}^{+}$is

$$
\tan \frac{\chi}{2} \simeq \frac{\left|\int_{-\frac{1}{\bar{v}}}^{0} \frac{-q E_{\perp}^{\prime \prime} \gamma_{\mathrm{r}}}{m_{\mathrm{e}}} \mathrm{d} t\right|}{\bar{v}}=\frac{p_{\perp} \gamma_{\mathrm{r}}}{p} \frac{l}{\left(l^{2}+\gamma_{\mathrm{r}}^{2} p^{2}\right)^{1 / 2}}(0<\chi \ll 1)
$$

where,

$$
p_{\perp}=\frac{q^{2}}{4 \pi \varepsilon_{0} m_{\mathrm{e}} \bar{\nu}^{2}} .
$$

